\title{
Genetic proclivities of two-component modulated aerobiosis
}

\author{
Hamzat Ibiyeye Tijani ${ }^{1, ~ *}$, Idris Abdulrahman ${ }^{2}$, Bashir Mohammed Abubakar ${ }^{3}$, \\ Sulaiman Mohammed ${ }^{4}$, Jibrin Ndejiko Mohammed ${ }^{5}$, Haruna Saidu ${ }^{6}$, Hindatu Yusuf ${ }^{7}$ \\ ${ }^{1}$ Faculty of Bioscience \& Medical Engineering, Universiti Teknologi Malaysia, 81310 Skudai, Johor-Bahru, Malaysia \\ ${ }^{2}$ Department of Microbiology, Kaduna State University, P.M.B. 2339, Kaduna \\ ${ }^{3}$ Department of Biological Sciences, Bauchi State University Gadau, P.M.B 065, Bauchi, Nigeria \\ ${ }^{4}$ Department of Biological Sciences, Gombe State University, PMB 0127, Gombe, Nigeria \\ ${ }^{5}$ Department of Microbiology, Ibrahim Badamasi Babangida University Lapai, P.M.B 011, Niger, Nigeria \\ ${ }^{6}$ Department of Biological Sciences, Gombe State University, PMB 0127, Gombe, Nigeria \\ ${ }^{7}$ Department of Biochemistry, Bauchi State University Gadau, P.M.B 065, Bauchi, Nigeria
}

\section{Email address:}

janyzrux@gmail.com (H. I. Tijani), abdurrahman.idris@kasu.edu.ng (I. Abdulrahman), elbash1150@yahoo.com (BM. Abubakar), sumulsu@yahoo.com (S. Mohammed),ndejiko@gmail.com(JN. Mohammed), saiduharunn@yahoo.com (H. Saidu), yusufhindat@yahoo.com (H. Yusuf)

\section{To cite this article:}

Hamzat Ibiyeye Tijani, Idris Abdulrahman, Bashir Mohammed Abubakar, Sulaiman Mohammed, Jibrin Ndejiko Mohammed, Haruna Saidu, Hindatu Yusuf. Genetic Proclivities of Two-Component Modulated Aerobiosis. Computational Biology and Bioinformatics. Vol. 2, No. 1, 2014, pp. 1-6. doi: 10.11648/j.cbb.20140201.11

\begin{abstract}
Great advances have been made in the past five decades in understanding the molecular mechanics of the two-component signal transduction pathway in bacteria but its applications in Medicine and Food Industries are yet to be fully unravelled. We discuss the varying changes in the extracellular environment of bacteria and their possession of multiple Two-Component Systems with each being specialize to react to a specific environmental signal, such as $\mathrm{pH}$, nutrient level, redox state, osmotic pressure, quorum signals, and antibiotics. The sensitivity of this response transmits information between different Two-Component Systems to form a complex signal transduction network. Bacteria's signal transduction system, referred to as a two-component system, are essential for adaptation to external stimuli. These systems provides a signal transduction pathways widely employed from prokaryotes to eukaryotes. Typically, each two-component system composed of a sensor protein distinctively monitors an external signal(s) and a response regulator (RR) that controls gene expression and other physiological activities which are collectively assembled in a signal transduction pathway. This annex reviews the molecular mechanics underlying the signal transduction systems in prokaryotic organisms. It is not uncommon to hear, either explicitly or implicitly, the statement that "two component regulatory systems are well understood". Therefore, we examine the current models of the mechanisms of the regulatory systems and provide viable suggestions to further expand its applications in drug efficiency and antibiotic resistance in humans as well as enhancing the shelf-life of products in the food industry. We also outline the challenges that might have quenched possible trials of this application to human health.
\end{abstract}

Keywords: Signal Transduction, Two-Component Arc System, Aerobiosis, Antibiotics

\section{Introduction}

With varying changes in the extracellular environment, bacteria possesses multiple Two-Component Systems with each being specialize to react to a specific environmental signal, such as $\mathrm{pH}$, nutrient level, redox state, osmotic pressure, quorum signals, and antibiotics. The sensitivity of this response transmits information between different Two-Component Systems to form a complex signal transduction network $[1,2]$.
Bacteria's signal transduction system, referred to as a two-component system, are essential for adaptation to external stimuli. These systems provides a signal transduction pathways widely employed from prokaryotes to eukaryotes. Typically, each two-component system composed of a sensor protein that distinctively monitors an external signal(s) and a response regulator (RR) that controls gene expression or other physiological activities such as 
chemo-taxis, which are collectively assembled in a signal transduction pathway via a phospho-relay system [3].

\section{Mechanisms of Signal Transduction}

Several studies have proposed different mechanisms for cellular signaling across biological membranes by receptors that possess an external ligand- binding sensory domain connected to a cytosolic catalytic domain. These mechanisms include changes in the structural conformation of the functional dimer by reversible protein phosphorylation (piston mechanism) or by rotational movement of the cytosolic domain. Although the rotational model is yet to be demonstrated experimentally, however, the piston model was apparent from studies on Tar, the methyl-accepting chemo-taxis protein for sensing aspartate in E. coli [4].

The piston mechanism were also found to operate viably in membrane-bound sensor kinases of two-component signal transduction systems and has been demonstrated for the bacterial EnvZ protein, a typical sensor kinase exhibiting only one cytoplasmic transmitter domain [5].

Two-component signal transducers, which are characterized by the histidine-aspartate phosphotransfer mechanism, were initially hypothesized to be restricted to prokaryotes; emphatic studies have now identified their activities in diverse eukaryotic species including plant, fungus, yeast and slime mold. In yeast, a two-component osmo-sensor has been found to regulate a mitogen-activated protein kinase (MAPK) cascade, a pervasive eukaryotic signaling component [6].

The enzymes that catalyze protein phosphorylation usually referred to as Protein kinases can be categorized based on the specific substrate-amino acid residues they phosphorylate: namely, protein serine/threonine kinases (PSKs); protein tyrosine kinases (PTKs); protein histidine kinases (PHKs); and also, a small sub-family of PSKs can phosphorylate both serine/threonine and tyrosine residues. Protein serine kinases and Protein tyrosine kinases are structurally related to each other as they originate from a common ancestral enzyme and were believed exist exclusively in eukaryotic cells, whereas Protein histidine kinases are a distinct family of enzymes, thought to be restricted to prokaryotes. However, recent studies disprove these differences as a family of PSKs with structural similarity to the mammalian PSKs was found in the prokaryote Myxococcus, and PHKs have been analyzed in diverse eukaryotic forms, including plant, fungus, yeast and slime mold [7].

Two-component regulatory systems (also called the HAP or His-Asp phosphotransfer mechanism) are widely used signaling machinery of bacterial adaptive responses. The system constitutes of sensor kinases and response regulators that can be phosphorylated and dephosphorylated by sensor kinases [8]. The representation of Figure 1 describes the general mechanism of the two component regulation.

Unlike archaea and eukaryotes, bacteria utilizes the two-component system as its major mode of signal transduction with most of the sensor being membrane-associated Histidine Kinase (HK). This sensor protein phosphorylates its own conserved histidine residue in response to a signal(s) in the environment. The carboxyl-terminal cytoplasmic region of Histidine kinase, called Transmitter domain, comprises of an ATP-binding domain and a so-called H-box domain that includes the conserved histidine residue for auto-phosphorylation [9].

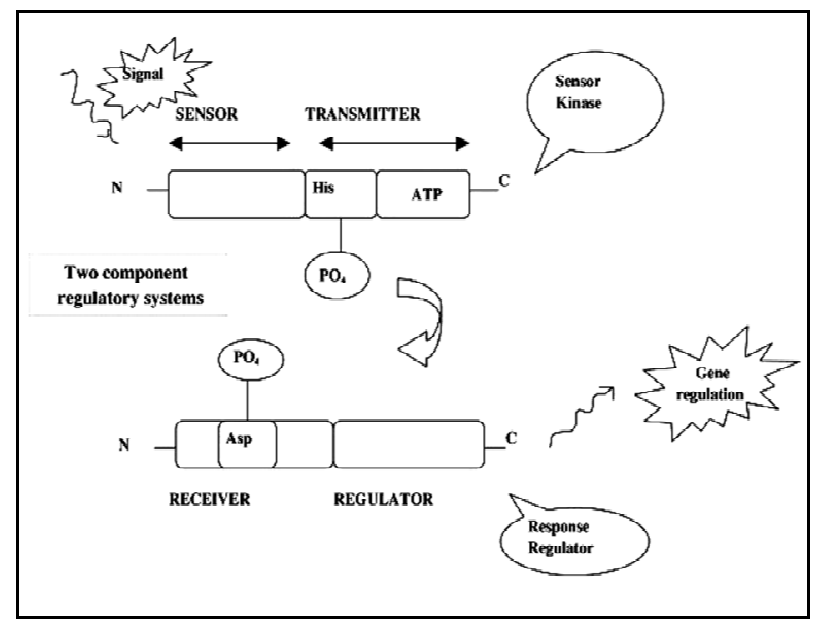

Figure 1. Mechanism of two-component regulatory systems

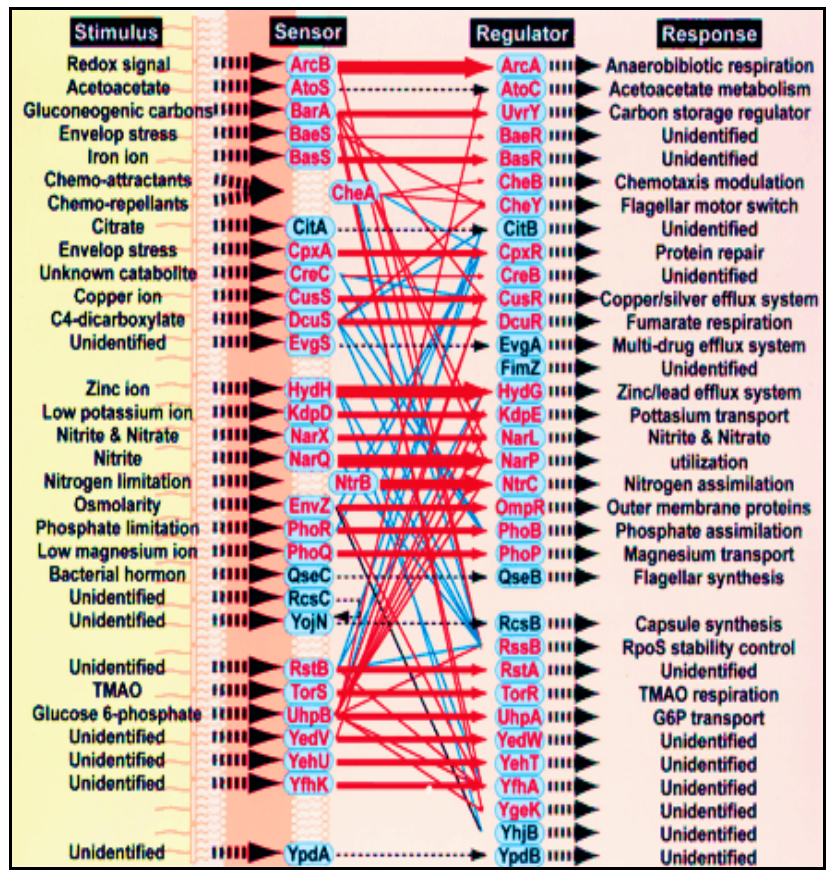

Figure 2. Cross-talk in trans-phosphorylation between non-cognate Histidne Kinase-Response Regulator pairs in Escherichia coli two-component systems (TCS)

Subsequently, the His-bound phosphoryl group of Histidine kinase is transferred onto a specific Aspartate (Asp) residue on the cognate response regulator (RR) for activation. The activated response regulator (RR) triggers, in most cases, the transcription of a set of genes, which respond to the external signal. On the basis of Escherichia coli 
genome sequence, a total of 30 Histidine kinases, each containing the conserved auto-phosphorylation domain, including yet uncharacterized putative HKs (BaeS, BasS, CreC, CusS, HydH, RstB, YedV, \& YfhK) and a total of 34 Response Regulators, each containing the conserved receiver domain, including putative RRs (BaeR, BasR, CreB, CusR, HydG, RstA, YedW, YfhA, YgeK, and YhjB) have been hypothesized to exist in Escherichia coli as illustrated in Figure 2 [10].

\section{Aerobiosis System - Escherichia Coli}

Signal transduction in biological systems typically involves receptor proteins that possess an extracytosolic sensory domain connected to a cytosolic catalytic domain. The ArcBA (anoxic redox control) system is a two component system in Escherichia coli that functions as the aerobiosis-sensing device that modulates the activity of the transcriptional regulation of oxidative and fermentative catabolic pathways to variations in oxygen availability. It regulates the transcription of more than 30 operons based on the redox conditions of growth; this system comprises of two components, the first component ArcB, a transmembrane sensor kinase functions as the sensor that relays a signal via signal-dependent kinase activity to the second component, ArcA which serves as the cognate response regulator (Figure 3) [11].

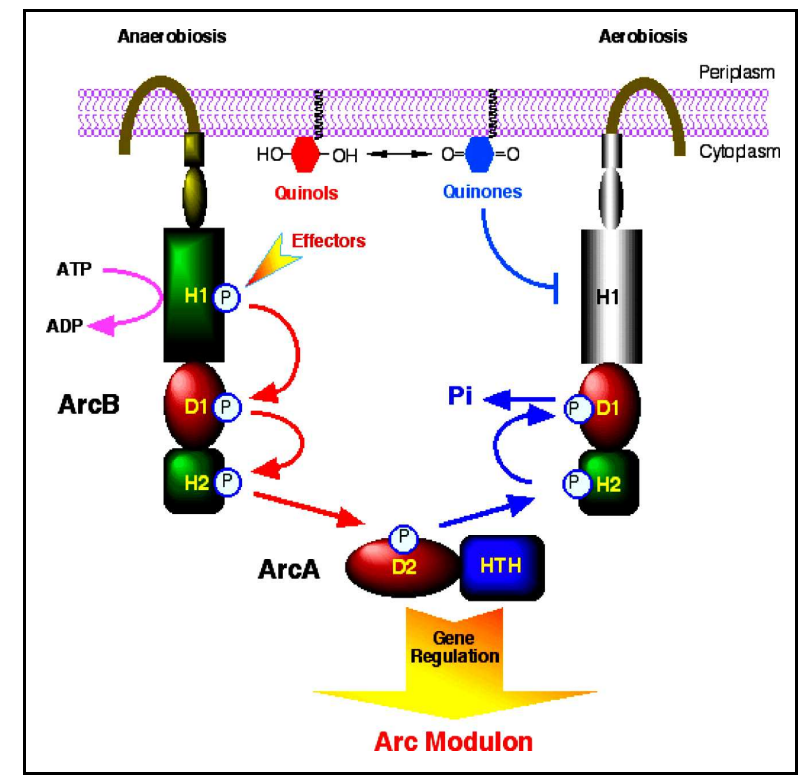

Figure 3. Phosphotransfer mechanism of ArcB sensor kinase

The ArcB sensor kinase of E. coli is a transmembrane protein without a significant periplasmic domain; it is unique in possessing an elaborate cytosolic structure that connects three catalytic domains: a primary transmitter with a conserved histidine residue at position 292, a receiver with a conserved aspartate residue at position 576, and a secondary transmitter with a conserved histidine residue at position 717. However, ArcA is a typical response regulator possessing an $-\mathrm{NH}_{2}$ terminus receiver domain with a conserved aspartate residue at position 54 and a $-\mathrm{COOH}$ terminus helix-turn-helix DNA binding domain [6].

\section{ArcBA system - Signal Transduction}

Under reducing conditions (absence of oxygen), the ArcB sensor protein undergoes autophosphorylation at His292, a reaction that is enhanced by several fermentative metabolites such as D-lactate, pyruvate, and acetate. The phosphoryl group is then sequentially transferred to ArcA via a His292 Asp576 His717 Asp54 phospho-relay system. Phosphorylated ArcA (ArcA-P), in turn, represses the transcription of many operons involved in respiratory metabolism and activates operons that encodes the synthesis of proteins and catabolic enzymes involved in fermentative metabolism; thus, optimal kinase activity of ArcB with ArcA is experienced under anaerobic conditions [11].

In the presence of oxygen (oxidizing conditions), the ArcB sensor protein autophosphorylation halts and the protein catalyzes the dephosphorylation of ArcA-P via an Asp54 His717 Asp576 reverse phospho-relay system. Consequently, the decreased level of phosphorylated ArcA induces the synthesis of some enzymes, such as the citric acid cycle enzymes, succinate dehydrogenases, lactate dehydrogenase, fumarase, pyruvate dehydrogenase, and the low oxygen affinity cytochrome $\mathrm{O}$ oxidase, while it deactivates the expression of other enzymes such as cytochrome D oxidase and enzymes involved in fermentative metabolism. The degree of dephosphorylation of ArcA determines the repression of operons involved in a wide variety of catabolic pathways that are operative under different redox growth conditions [12,13,14]. Thus, the ArcBA system is important for the organism's ability to distribute energy generation for fermentation and respiration conditions.

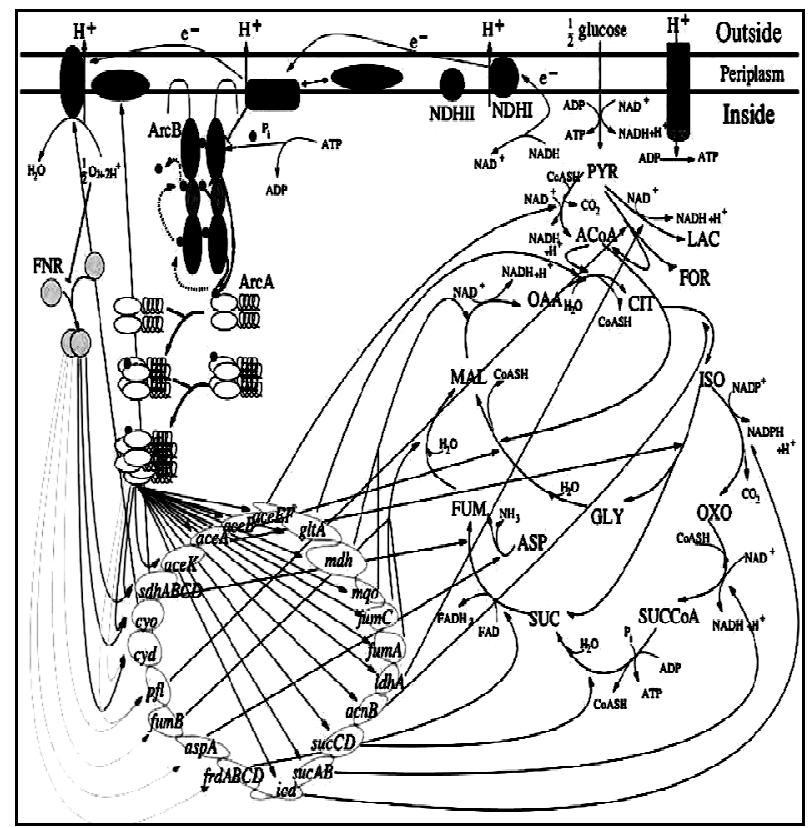

Figure 4. Signal transduction of two-component Arc systems 
Figure 4 depicts the schematic diagram showing the activity of ArcB autophosphorylation in relation to aerobic and anaerobic extracellular environment and the effect of ubiquinone and menaquinone cytosolic pool on signal transduction in Escherichia coli. Oxidized forms of quinone electron carriers behave as inhibitory signals that inhibits ArcB autophosphorylation. Fermentative metabolites such as D-lactate, pyruvate, and acetate behave as effectors that promote ArcB autophosphorylation.

\section{ArcB Protein - Membrane Interaction}

Contradictory to the structural feature of typical sensor kinases that have a substantial periplasmic domain, the ArcB has a short periplasmic sequence of only about 16 amino acid residues for environmental sensing. Studies have shown that replacing various segments within the ArcB membrane region by a counterpart of MalF (a subunit of maltose permease without any sequence homology with ArcB) reveals no significant effect on the signal transduction process thus, the ArcB domain bounded by the two transmembrane segments (amino acid residues 22-77) does not directly interact in signal recognition but rather serves as an anchor that affix the protein to the membrane matrix close to the source of the signal $[15,16]$.

ArcB can form intermolecular disulfide bridges via Cys-180 and Cys-241, which are located in the "PAS domain" of the protein; the kinase activity of ArcB is highly dependent on this covalent linkage. The PAS domain is a unique subunit of a protein that serves as the signal sensor in many signaling proteins found in wide array of organisms from bacteria to humans. This domain was assigned this attribute "PAS" after three proteins in which it is inherent:

- Per - period circadian protein

- Arnt - aryl hydrocarbon receptor nuclear translocator

- Sim-single-minded protein

A disulfide linkage formed between two Cys- 180 residues of the ArcB sensor protein results in an $85 \%$ reduction in kinase activity, while those formed between two Cys-241 residues results in a $15 \%$ reduction in the kinase activity of ArcB sensor protein [17].

\section{Ubiquinone/Menaquinone - Redox}

Earlier in vitro experimental studies postulates that the redox state of the ubiquinone pool is the determinant for ArcB kinase activity. However, it was later discovered that upon deletion of a ubiquinone biosynthetic enzyme, regulation of $\mathrm{ArcB}$ in the anaerobic-aerobic transition is not affected. In contrast, interference with menaquinone biosynthesis results in the inactivation of $\mathrm{ArcB}$ sensor kinase during anaerobic growth; this inactivity can fully be restored by addition of a menaquinone precursor. This vividly proves that menaquinones play a major role in ArcB activation as ArcB is activated under anaerobic and sub-aerobic conditions and is much less active under fully aerobic and micro-aerobic conditions [18].
In addition, there is no interaction between $\mathrm{ArcB}$ sensor kinase activation and the redox state of the ubiquinone pool, with exception to the fact that a restricted communication exists between the total cellular ubiquinone concentration and ArcB activity because a substantial increase in the concentration of the ubiquinone pool will increase the degrees of aerobiosis; . These observations proves the mechanistic hypothesis that the in vivo activity of ArcB in $E$. coli is modulated by the redox state of the menaquinone pool and that the ubiquinone/ubiquinol ratio in vivo surely is not the only determinant of ArcB activity [19].

\section{Bacterial Antibiotic Resistance}

Antibiotics, also known as antibacterials, are types of medications that destroy or slow down the growth of bacteria life forms; they were known to be one the great discoveries of the $20^{\text {th }}$ century. However, the application of this class of drug has been hampered by the onset of new antimicrobial resistant bacterial strains. The first clinical antibiotic, penicillin, was introduced in the mid-1940s, while the existence of resistant mutants were acknowledged within 2 years of its introduction [20].

The histidine kinase sensor protein, which is phosphorylated on a conserved histidine residue, transfers the phosphoryl group to the aspartic acid of the response regulator. Many phosphorylated response regulators bind to the upstream-regulatory region of transcription unit of the pathogenic genes and control their expression. The phosphorylated response regulators (RR-P) is sometimes also dephosphorylated by the histidine kinase (HK) depending on the signaling response of the perisplamic domain of the sensor protein; thus the phosphorylation state of the response regulators within the cytoplasm is determined by the balance between phosphorylation and dephosphorylation, and this in turn controls gene expression [21].

Some Two-Component Systems also control gene clusters that contribute to cell growth, virulence, biofilms, quorum sensing etc. Conventional antibiotics are specific and acts distinctively to alter the activities of target proteins that carry out vital cellular functions, whereas drugs that specifically inhibit Two-Component Systems (TCS) target the upstream regulatory control functions, not the downstream activities. Thus, anti-TCS drugs exact its inhibitory role in a manner quite different from existing drugs' action mechanism; the development of these anti-TCS drugs and pesticides are effective against various drug-resistant bacteria. In other words, drugs that target a Two-Component System required for growth may serve as new antibiotics that can kill multi-drug-resistant bacteria such as methicillin-resistant Staphylococcus aureus (MRSA) and vancomycin-resistant Enterococcus (VRE) [22,23,24].

Furthermore, inhibitors of Two-Component Systems that control virulence factors, such as biofilms and quorum sensing (QS), could control virulence without killing the pathogenic bacteria $[25,26]$. Two-Component Systems are 
also involved in the pathogenicity of certain eukaryotes and it is believed that the TCSs in medically important fungal pathogens are considered as potential drug targets [27].

To date, anti-TCS drugs that repress virulence in most pathogen organisms have specifically targeted the sensory domains of their target histidine kinases. Unlike the conserved catalytic domains of histidine kinases, the sensory domains are unique to each histidine kinase, thus limiting the affinity of sensory domain-targeting drugs effective only to a specific Two-Component System [28].

Currently, one of the most crucial complications being encountered is the resistive features of bacterial strains to Vancomycin, a glycopeptide antibiotics used in the prophylaxis and treatment of infections caused by Gram-positive bacteria; it is the antibiotics often used as the last treatment option. Also, the signals transmitted and detected by sensory proteins are still unclear in many of the Two-Component Systems involved in virulence, and there are various cases where signals from multiple Two-Component Systems are integrated to coordinate a single cell response [28,29].

Below is a mechanistic representation of the inhibitory role of anti-TCS drugs on the transcriptional process in pathogenic organisms.

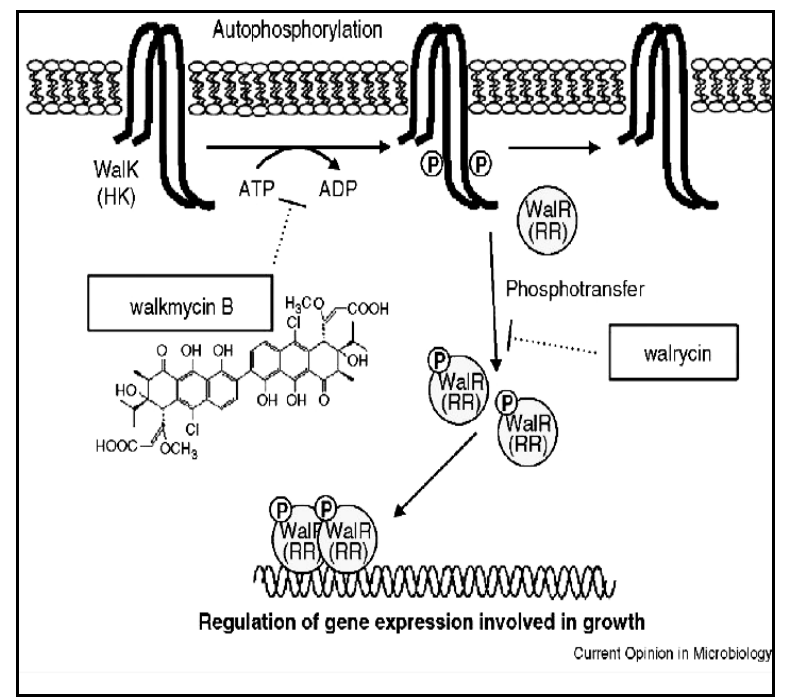

- Walkmycin B is the first antibiotic targeting WalK histidine kinase

- Walrycins are the first inhibitors targeting WalR response regulator

Figure 5. Inhibitors targeting the essential Two-Component System, WalK/WalR. (Walkmycin B blocks autophosphorylation of WalK; Walrycin interacts with WalR and blocks phosphotransfer from WalK)

\section{References}

[1] Eguchi Y, Utsumi R: Introduction to bacterial signal transduction networks. Adv Exp Med Biol 2008, 631:1-6.

[2] Mitrophanov AY, Groisman EA: Signal integration in bacterial two-component regulatory systems. Genes Dev 2008, 22:2601-2611

[3] Mark Goulian: Two-component signaling circuit structure and properties. Current Opinion in Microbiology 2010, 13:184-189 DOI 10.1016/j.mib.2010.01.009.

[4] Ohsuk Kwon , Dimitris Georgellis, and Edmund C. C. Lin: Rotational On-off Switching of a Hybrid Membrane Sensor Kinase Tar-ArcB in Escherichia coli . Journal of Biological Chemistry Vol. 278, No. 15, pp. 13192-13195, 2003 DOI 10.1074/jbc.M210647200

[5] Marie Foussard, Stéphanie Cabantous, Jean-Denis Pédelacq, Valérie Guillet, Samuel Tranier, Lionel Mourey, Catherine Birck, Jean-Pierre Samama: The molecular puzzle of two-component signaling cascades. Microbes and Infection, $3,2001,417-424$.

[6] Luchi, A. S., and Lin, E. C. C. (1996): Escherichia coli and Salmonella typhimurium: Cellular and Molecular biology pp. 1526-1538, American Society for Microbiology, Washington, D. C.

[7] Hoch, J.A: Two-component and phosphorelay signal transduction. Curr Opin Microbiol 2000, 3:165-70.

[8] Stock JB, Ninfa AJ, Stock AM: Protein phosphorylation and regulation of adaptive responses in bacteria. Microbiol Rev 1989, 53:450-490.

[9] Masayuki Matsushita, Kim D. Janda: Histidine Kinases as Targets for New Antimicrobial Agents. Bioorganic \& Medicinal Chemistry 10 (2002) 855-867.

[10] Yamamoto K, Hirao K, Oshima T, Aiba H, Utsumi R, Ishihama A (2005). "Functional characterization in vitro of all two-component signal transduction systems from Escherichia coli." J Biol Chem 280(2); 1448-56. PMID: 15522865 .

[11] Luchi S. and Lin E. C. (1993): Adaptation of Escherichia coli to redox environments by gene expression. Mol. Microbiol. 9:9-15.

[12] Alexeeva, S., K. J. Hellingwerf, and M. J. Teixeira de Mattos. 2003. Requirement of ArcA for redox regulation in Escherichia coli under microaerobic but not anaerobic or aerobic conditions. J. Bacteriol. 185:204-209.

[13] Govantes, F., J. A. Albrecht, and R. P. Gunsalus. 2000. Oxygen regulation of the Escherichia coli cytochrome d oxidase (cydAB) operon: roles of multiple promoters and the Fnr-1 and Fnr-2 binding sites. Mol. Microbiol. 37:1456-1469.

[14] Liu, X., and P. De Wulf. 2004. Probing the ArcA-P modulon of Escherichia coli by whole genome transcriptional analysis and sequence recognition profiling. J. Biol. Chem. 279:12588-12597.

[15] Georgellis, D., A. S. Lynch, and E. C. Lin. 1997. In vitro phosphorylation study of the arc two-component signal transduction system of Escherichia coli. J. Bacteriol. 179:5429-5435.

[16] Kwon, O., D. Georgellis, and E. C. Lin. 2000. Phosphorelay as the sole physiological route of signal transmission by the arc two-component system of Escherichia coli. J. Bacteriol. $182: 3858-3862$.

[17] Malpica, R., B. Franco, C. Rodriguez, O. Kwon, and D. Georgellis. 2004. Identification of a quinone-sensitive redox switch in the ArcB sensor kinase. Proc. Natl. Acad. Sci. U. S. A. 101:13318-13323. 
[18] Sawers, Joost Teixeira de Mattos and Klaas Hellingwerf Martijn Bekker, Svetlana Alexeeva, Wouter Laan, Gary: The ArcBA Two-Component System of Escherichia coli is regulated by the Redox State of both the Ubiquinone and the Menaquinone Pool. J. Bacteriol. 2010, 192(3):746. DOI: 10.1128/JB.01156-09.

[19] Alvarez AF, Georgellis D. (2010): In vitro and in vivo analysis of the ArcB/A redox signaling pathway. Methods Enzymol. 2010; 471:205-28. Epub 2010 Mar 1.

[20] Medicinal News Today, 2009: What Are Antibiotics? How Do Antibiotics Work? Microbiol. Rev. 32, 461-473 http://www.medicalnewstoday.com/articles/10278.php

[21] Yasuhiro Gotoh, Yoko Eguchi, Takafumi Watanabe, Sho Okamoto, Akihiro Doi and Ryutaro Utsumi: Two-component signal transduction as potential drug targets in pathogenic bacteria. Current Opinion in Microbiology 2010, 13:232-239 DOI 10.1016/j.mib.2010.01.00.

[22] Dubrac S, Msadek T: Tearing down the wall: peptidoglycan metabolism and the WalK/WalR (YycG/YycF) essential two component system. Adv Exp Med Biol 2008, 631:214-228.

[23] Rasko DA, Moreira CG, Li de R, Reading NC, Ritchie JM, Waldor MK, Williams N, Taussig R, Wei S, Roth Met al.: Targeting QseC signaling and virulence for antibiotic development. Science 2008, 321:1078-1080.
[24] Watanabe T, Okada A, Gotoh Y, Utsumi R: Inhibitors targeting two-component signal transduction. Adv Exp Med Biol 2008, 631:229-236.

[25] Cegelski L, Marshall GR, Eldridge GR, Hultgren SJ: The biology and future prospects of anti-virulence therapies. Nat Rev Microbiol 2008, 6:17-27.

[26] Dong YH, Wang LH, Xu JL, Zhang HB, Zhang XF, Zhang LH: Quenching quorum-sensing-dependent bacterial infection by an $\mathrm{N}$-acyl homoserine lactonase. Nature 2001, 411:813-817.

[27] Chauhan N, Calderone R: Two-component signal transduction proteins as potential drug targets in medically important fungi. Infect Immun 2008, 76:4795-4803.

[28] Yoko Eguchi, Norihiro Kubo, Hiroko Matsunaga, Masayuki Igarashi and Ryutaro Utsumi: Development of an Antivirulence Drug against Streptococcus mutans: Repression of Biofilm Formation, Acid Tolerance, and Competence by a Histidine Kinase Inhibitor, Walkmycin C Antimicrob. Agents Chemother. 2011, 55(4):1475. DOI: 10.1128/AAC.01646-10.

[29] Mohammed J.N, Abubakar B.M, Yusuf H., Sulaiman M., Saidu H., Idris A., Tijani H.I. Bacterial Biofilm: A Major Challenge of Catheterization. J. of Microbiology Res. 2013, 3(6) : 213 - 223 DOI:10.5923/j.microbiology.20130306.04 